\title{
Automatic Identification and Segmentation of Diffuse Retinal Thickening Macular Edemas Using OCT Imaging ${ }^{\dagger}$
}

\author{
Gabriela Samagaio ${ }^{1,2}$, Joaquim de Moura ${ }^{1,2, *}$, Jorge Novo ${ }^{1,2}$ and Marcos Ortega 1,2 \\ 1 Department of Computing, University of A Coruña, 15071 A Coruña, Spain; \\ gabriela.samagaio@udc.es (G.S.); jnovo@udc.es (J.N.); mortega@udc.es (M.O.) \\ 2 CITIC-Research Center of Information and Communication Technologies, University of A Coruña, \\ 15071 A Coruña, Spain \\ * Correspondence: joaquim.demoura@udc.es; Tel.: +34-981167000 \\ + Presented at the XoveTIC Congress, A Coruña, Spain, 27-28 September 2018.
}

Published: 18 September 2018

\begin{abstract}
This paper proposes a novel methodology for the automatic identification and segmentation of the Diffuse Retinal Thickening (DRT) edemas using Optical Coherence Tomography (OCT) images as source of information. This Macular Edema (ME) type is commonly used by ophthalmologists as a relevant biomarker for the early diagnosis of this retinal disorder which, therefore, permits a better adjustment of the treatments, reducing their costs as well as improving the life quality of the patients.
\end{abstract}

Keywords: computer-aided diagnosis; Optical Coherence Tomography; Diffuse Retinal Thickening region; segmentation

\section{Introduction}

Diabetic retinopathy is one of the leading causes of vision impairment that affects $1 \%$ of the world population [1]. Diffuse Retinal Thickening (DRT) is a Macular Edema (ME) type derived from the local intraretinal fluid accumulation in the lower retinal layers. As illustrated in Figure 1, the presence of this edema produces profound structural and morphological modifications in the eye fundus, as an increment of the lower retinal layers. The absence of a limiting membrane allows the fluid to spread over the outer retinal region, leading to a single and continuous region.

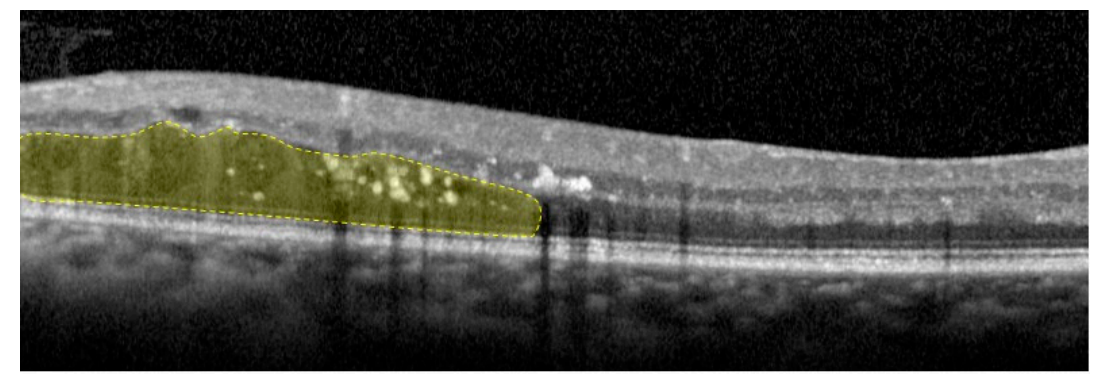

Figure 1. Example of OCT image with the DRT edema presence.

To identify the presence of retinal disorders, Optical Coherence Tomography (OCT) imaging is being widely used within the ophthalmological community. Moreover, it offers an easy visualization of the in vivo histopathology of the retina in a contactless and non-invasive capture process. 
In this paper, we propose a new methodology for the automatic identification and segmentation of the DRT presence in OCT images using as reference the clinical classification [2,3]. The precise localization and delimitation of the DRT allow the early diagnosis of this disease and consequently, it permits a better adjustment of the treatments, reducing their costs as well as improving the life quality of the patients.

\section{Methodology}

The proposed methodology is composed by three main stages for the identification and segmentation of DRT edemas [4]. Firstly, the system segments the retinal layers to facilitate the search of this ME type in the Region of Interest (ROI) where they typically appear, the outer retina. Secondly, a learning strategy is applied to identify the DRT presence and segment its constituent region. Finally, two post-processing strategies are implemented and tested to individually refine the impact of the False Positives (FPs) and the False Negatives (FNs) detected regions from the classifier output and improve the obtained results.

\section{Results and Conclusions}

The proposed methodology achieved satisfactory results for the automatic identification and segmentation of the DRT edemas. Using the best classifier configuration, we applied two individuals post-processing strategies to improve the obtained results. Figure 2 illustrates the resulting image provided by the second post-processing, as the best configuration. This strategy unifies nonconsecutive DRT regions, improving significantly the efficiency of the system and consequently facilitating the identification and visualization of the area affected by this pathology.

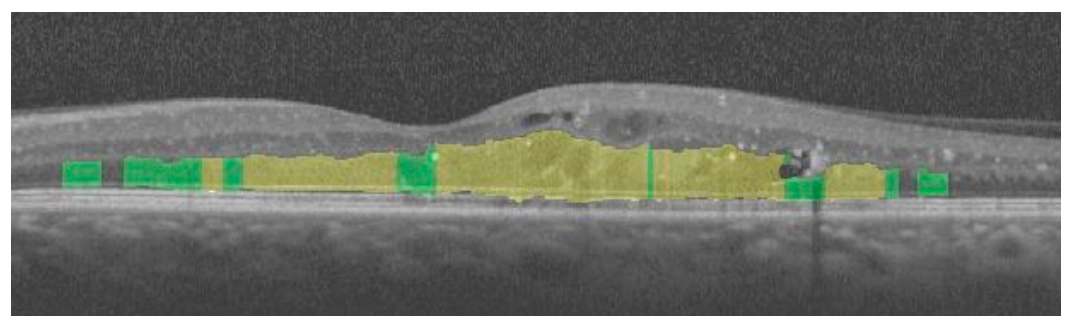

Figure 2. Illustrative output OCT image after the application of the second post-processing approach. Yellow regions, direct results from the classifier. Green regions, results of the second post-processing approach.

Author Contributions: G.S., J.d.M. and J.N. contributed to the development of the designed methodology and the analysis of the experimental evaluation methods. M.O. supported with the domain-specific knowledge. All the authors supervised and analyzed the obtained results. G.S. was responsible for writing the manuscript. All the authors cooperated in the critical revision and final approval of the manuscript structure.

Acknowledgments: This work is supported by the Instituto de Salud Carlos III, Government of Spain and FEDER funds of the European Union through the PI14/02161 and the DTS15/00153 research projects and by the Ministerio de Economía y Competitividad, Government of Spain through the DPI2015-69948-R research project. Also, this work has received financial support from the European Union (European Regional Development Fund-ERDF) and the Xunta de Galicia, Centro singular de investigación de Galicia accreditation 2016-2019, Ref. ED431G/01; and Grupos de Referencia Competitiva, Ref. ED431C 2016-047.

Conflicts of Interest: The authors declare no conflict of interest. The founding sponsors had no role in the design of the study; in the collection, analyses, or interpretation of data; in the writing of the manuscript, and in the decision to publish the results 


\section{References}

1. World Health Organization. World Health Statistics 2010; World Health Organization: Geneva, Switzerland, 2010.

2. Otani, T.; Kishi, S.; Maruyama, Y. Patterns of diabetic macular edema with optical coherence tomography. Am. J. Ophthalmol. 1999, 127, 688-693.

3. Panozzo, G.; Parolini, B.; Gusson, E.; Mercanti, A.; Pinackatt, S.; Bertoldo, G.; Pignatto, S. Diabetic macular edema: An OCT-based classification. Semin. Ophthalmol. 2004, 19, 13-20.

4. Samagaio, G.; de Moura, J.; Novo, J.; Ortega, M. Automatic segmentation of diffuse retinal thickening edemas using Optical Coherence Tomography images. Procedia Comput. Sci. 2018, 126, 472-481.

(C) 2018 by the authors. Licensee MDPI, Basel, Switzerland. This article is an open access article distributed under the terms and conditions of the Creative Commons Attribution (CC BY) license (http://creativecommons.org/licenses/by/4.0/). 